\title{
Motivational interviewing for improving periodontal health
}

\author{
Siebert T, Malachovsky I, Statelova D, Stenchlakova B \\ Department of Stomatology and Maxillofacial Surgery, Jessenius Faculty of Medicine in Martin, Comenius \\ University in Bratislava, University Hospital in Martin, Slovakia. b.stenchlakova@gmail.com
}

\begin{abstract}
Periodontal health should be defined as a state free from inflammatory periodontal disease that allows an individual to function normally and not suffer any consequences (mental or physical) as a result of past disease. Tissues located in the vicinity of implants, known as peri-implantation tissues, have specific and in many ways similar anatomy. The elaborate protocol of preventive measures to ensure periodontal health is extremely important. There is compelling evidence that motivational interviewing frequently leads to more favourable clinical responses than conventional preventive tools. Herein, we describe that the periodontal health can serve as a vital common reference point for assessing disease, determining meaningful preventive methods and treatment outcomes. This article mainly focuses on the role of new clinical data regarding periodontal health and motivational interviewing, which received a particular attention in recent years such as motivation for abidance and on the prognosis of periodontal health (Tab. 3, Ref. 26). Text in PDF www.elis.sk KEY WORDS: periodontal health, gingivitis, periodontitis, motivation, motivational interviewing.
\end{abstract}

\section{Introduction}

"Health is a state of complete physical, mental and social wellbeing and not merely the absence of disease or infirmity" (1). In accordance with this definition by the World Health Organization, periodontal health should be defined as a state free from inflammatory periodontal disease that allows an individual to function normally and not suffer any consequences (mental or physical) as a result of past disease (2). In daily practice, we encounter patients in whom we find diseases of the supporting tissue of the teeth, the periodontium. A healthy periodontal system is a key component of oral health and an important part of general health and subjective well-being at the individual and entire population levels. Healthy gingiva can be defined as the buffer system for teeth. Disease development usually begins in the gingiva. In addition to the gingiva and cement of the tooth itself, the periodontal structures include alveolar bone and periodontal fibres. Tissues located in the vicinity of implants, also known as peri-implantation tissues, have specific and in many ways similar anatomy.

According to recent epidemiological data, gingivitis affects up to $95 \%$ of the world's population, and chronic periodontitis

Department of Stomatology and Maxillofacial Surgery, Jessenius Faculty of Medicine in Martin, Comenius University in Bratislava, University Hospital in Martin, Slovakia

Address for correspondence: B. Stenchlakova, MDDr, Comenius University, Jessenius Faculty of Medicine, Department of Stomatology and Maxillofacial Surgery, Jessenius Faculty of Medicine in Martin, Comenius University in Bratislava, University Hospital in Martin, Kollarova 2, SK-036 01 Martin, Slovakia.

Phone: +421.907097456 affects up to $60 \%$ to $65 \%$ of the North American population 65 years and older $(3,4)$. In the study of more than 9402 patients, the incidence of periodontitis in the US was nearly half (45.9\%) the population aged 30 years and older affected. A better understanding of the factors influencing these findings and the disparities among socio-demographic groups is important for public health action to prevent and control periodontitis in US adults (4). The vast majority of periodontal and peri-implantation tissue diseases are initiated by the accumulation of oral microbiological biofilm on the hard and soft tissues of the teeth and oral cavity. This biofilm causes local inflammatory reactions in the marginal region of the gingival soft tissue and peri-implantation mucosa. If the bacterial biofilm is not adequately and regularly removed professionally by a dentist and individually by the patient through household oral hygiene measures, the biofilm becomes dysbiotic.

Even when dental plaque biofilm levels are minimized, an inflammatory infiltrate is present within gingival tissues as part of a physiologic immune surveillance (2). However, the initiation of gingivitis occurs if dental plaque accumulates over days or weeks without disruption or removal, due to a loss of symbiosis between the biofilm and the host's immune-inflammatory response, and development of an incipient dysbiosis (5). Various systemic factors, including endocrinopathies, hematologic conditions, diet, and drugs, can modify the immune-inflammatory response $(5,6)$.

Together with local and overall factors, conditions are created that promote the multiplication of pathogenic bacteria that, over time, lead to the development of chronic soft tissue inflammation such as gingivitis or mucositis of peri-implantation tissues. In susceptible individuals, the persistence of gingivitis leads to the development of periodontitis. In the case of a patient who also has 
Tab. 1. Classification of periodontal diseases and conditions (Caton et al, 2018).

\begin{tabular}{|c|c|c|c|c|c|}
\hline \multicolumn{6}{|c|}{ Classification of periodontal diseases and conditions } \\
\hline \multicolumn{6}{|c|}{ Periodontal Health Gingival Diseases and Conditions } \\
\hline $\begin{array}{l}\text { Periodontal Health and } \\
\text { Gingival Health } \\
\end{array}$ & \multicolumn{2}{|c|}{$\begin{array}{l}\text { Gingivitis: Dental } \\
\text { Biofilm - Induced }\end{array}$} & \multicolumn{3}{|c|}{$\begin{array}{c}\text { Gingival Diseases: Non-dental } \\
\text { Biofilm - Induced } \\
\end{array}$} \\
\hline \multicolumn{6}{|l|}{ Periodontitis } \\
\hline $\begin{array}{l}\text { Necrotising Periodontal } \\
\text { Diseases }\end{array}$ & \multicolumn{2}{|c|}{ Periodontitis } & \multicolumn{3}{|c|}{$\begin{array}{c}\text { Periodontitis as a Manifestation } \\
\text { of Systemic Diseases }\end{array}$} \\
\hline \multicolumn{6}{|c|}{ Other Conditions Affecting the Periodontium } \\
\hline $\begin{array}{l}\text { Systemic diseases or conditions } \\
\text { affecting the periodontal } \\
\text { supporting tissues }\end{array}$ & $\begin{array}{c}\text { Periodontal abscesses } \\
\text { and endodontic- } \\
\text { periodontal lesions }\end{array}$ & $\begin{array}{r}\text { Muc } \\
\text { defor } \\
\text { con }\end{array}$ & & $\begin{array}{c}\text { Traumatic } \\
\text { occlusal } \\
\text { forces }\end{array}$ & $\begin{array}{l}\text { Tooth and } \\
\text { prosthesis related } \\
\text { factors }\end{array}$ \\
\hline
\end{tabular}

adopted to cover the absence of (or very significant reduction in) clinical periodontal inflammation on either an anatomically intact periodontium or a reduced periodontium.

Good oral hygiene has always been considered a mainstay of periodontal health (2, 9). It is usually achieved by a combination of good personal oral hygiene and regular professional care. While oral hygiene remains the most important factor in obtaining and maintaining periodontal health, it should not be the sole focus of attention. Additional factors, such as motivational in-

implanted implant fixtures and prosthetic implants in his oral cavity, inflammation and damage to bone tissues around the implants may occur in addition to peri-implant mucositis. Periodontal health has not been defined in any of the classifications of periodontal diseases and conditions. Interestingly, there are almost minimum studies or reports attempting to define periodontal health $(2,7)$.

Rehabilitation of the orofacial area and reconstruction of the teeth are important for the patient not only for nutrition, aesthetics and phonation, but also for mental well-being.

Defining periodontal health is very important if we are to have a common reference point for assessing periodontal disease and determining meaningful treatment outcomes.

Health can be evaluated at both the histological and clinical levels and should be considered in the context of a preventive starting point and a therapeutic end point.

\section{Periodontal health}

A classification scheme for periodontal and peri-implantation diseases and conditions is necessary for clinicians to properly diagnose and treat patients as well as for scientists to investigate aetiology, pathogenesis, natural history, and treatment of diseases and conditions (8). This paper summarizes the proceedings of the World Workshop on the Classification of Periodontal and Peri-implantation Diseases and Conditions. This workshop was co-sponsored by the American Academy of Periodontology (AAP) and the European Federation of Periodontology (EFP) and included expert participants from all over the world. Planning for the conference, which was held in Chicago from November 9 to 11, 2017, began in early 2015 (8). This new classification represents the work of the worldwide community of scholars and clinicians in periodontology and implant dentistry. The result of this classification is also the newly created and worldwide valid definition of periodontal health (Tab. 1). This workshop also characterized periodontal health and gingival inflammation in a reduced periodontium after the successful treatment of a patient with periodontitis (Tab. 2).

In its pristine form, periodontal health would be defined as the absence of histological evidence of periodontal inflammation and no evidence of anatomical change to the periodontium. However, it must be recognized that in most (if not all) adults, this is unlikely (2). Therefore, the term clinically healthy should be terviewing, must be addressed in the quest for attaining or maintaining periodontal health.

\section{Motivational interviewing of the patient}

Current epidemiological data on the prevalence of gingivitis suffer from the lack of a universally adopted case definition and vary as widely as $6 \%$ to $94 \%$, due to the use of indices that measure gingival inflammation at individual sites rather than considering the patient's mouth as a whole. Therefore, mild localized clinical inflammation is reported to affect almost $95 \%$ of the population, a figure that would incorrectly suggest gingivitis as being a variation of "normality" and thus consistent with the spectrum of "clinical health" rather than being a disease (10).

Randall (2018) performed a study, that was evaluating the behavior as an important factor in dental disease etiology. So behavioral interventions are needed for prevention and treatment. Motivational interviewing has been proposed as a potentially useful behavioral intervention for oral health promotion, but results from published studies are mixed. Furthermore, this literature is immature; basic efficacy research and innovative applications are still needed. Although likely not as a stand-alone intervention, motivational interviewing may hold promise for dental public health (11).

Epidemiologic studies have revealed that smoking is one of the major lifestyle-related environmental risk factors for periodontal disease (5). Smoking has been proven to increase systemic inflammation in previous studies using different biomarkers (12). Although plaque accumulation and disease progression are exacerbated in smokers, smokers have fewer clinical signs and symptoms of gingival inflammation, and therefore smoking can mask an underlying gingivitis (5). The patient has a decisive influence on the prevention and therapy of periodontal diseases. We can even discuss a critical point for the success of periodontal health and the treatment of periodontal disease itself, from the appearance of the first symptoms, diagnosis, and treatment to supportive periodontal therapy.

Tab. 2. Periodontal and gingival health (Caton et al, 2018).

Periodontal Health and Gingival Health

Clinical gingival health on an intact periodontium

Clinical gingival health on a reduced periodontium

Stable periodontitis patient

Non-periodontitis patient 
$670-674$

A counseling approach, motivational interviewing (MI)., is potentially useful in changing oral health behaviors. In the study of Gao et al (2014) literature was searched to identify randomized controlled trials that evaluated the effectiveness of MI in changing oral health behaviors and improving oral health of dental patients and the public. Concerning periodontal health, superior effect of MI on oral hygiene was found in five trials and was absent in two trials. These reviewed randomized controlled trials showed varied success of MI in improving oral health. The potential of MI in dental health care, especially on improving periodontal health, remains controversial (13). Successful results can only be achieved if the patient cooperates actively. Treatment may not be successful for patients who are actively involved in the therapy process.

In a qualitative study, Curry-Chiu et al (2015) investigated supporting use of MI to change oral health behaviors in the areas of early childhood caries and periodontal diseases. Research was limited due to the sparse number of oral health care providers with training in MI. The aim of this qualitative study was to use semi-structured interviews with nine program alumni to provide insight into the experiences of MI-trained dental hygienists in clinical practice. All interviews were captured with a digital voice recorder, were transcribed, and were resubmitted to the interviewees for checking. Five themes emerged from the data analysis: salience, best practices, barriers, facilitators, and lessons learned. These dental hygienists strongly valued and embraced the spirit of MI. The participants approved of their MI instruction as a whole but felt it was difficult and sometimes not viable in practice. They reported that MI training had improved their communication skills and increased treatment acceptance. Time, difficulty, and managing patient resistance were the most often cited barriers, while a supportive climate and creating a routine were the most often cited facilitators (14).

Periodontal health is supported by appropriate behaviours such as regular self-performed plaque control, avoidance of tobacco, and glycaemic control with diabetes mellitus type 2 (15). Research into the prevention and control of periodontal diseases considers both the general population and the individual. Children, young people, adults and older patients are monitored and supported; each of these groups has its own particularities and issues. The results from the national YRBSS (Youth Risk Behavior Surveillance System) in 2017 indicated that many high school students are engaged in health-risk behaviours (16). These risk behaviours are established during childhood and adolescence and extend into adulthood $(16,17)$.

Accordingly, an oral health intervention was developed using MI, a widely accepted guiding style for enhancing intrinsic motivation to change with worldwide dissemination and application in a variety of disciplines. While use of MI for oral health concerns is more nascent and minimally studied in indigenous groups, the technique is consistent with cultural values of respect, autonomy and honouring the individual's wisdom (18). Overall, it would be very effective if the governments were involved in the prevention of periodontal diseases, both at the national level and at the regional level. Where declining preventive action is observed, local education needs to be supplemented. In particular, the den- tal community is able to act preventively and therapeutically to provide health care for individuals or groups located within the appropriate catchment area and, for dental practitioners, in the area in which patients most frequently visit, such as for specialist appointments. Dentists, if they want to be effective in motivating prevention, should also understand the relationship between the patient's health status and the effects of inappropriate patient behaviour in terms of taking home care measures.

One of the most important aspects of oral health that individuals can control is household oral hygiene. Good oral hygiene has always been considered the foundation in the treatment of periodontal diseases. The review of the authors Kay et al (2016) included eight papers all of which were considered to be of robust quality, in terms of their research methods and seven of which were considered to offer externally valid findings. Five described randomised controlled trials and all of these RCTs demonstrated that interventions including motivational interviewing had a positive effect on oral health and health behaviour. This review shows that the motivational interviewing technique, which is based on the concept of autonomy support, has a potential for helping patients with poor oral health. Training in motivational interviewing for dental personnel could be a very useful addition to the skill set of practitioners and dental teams (19).

Usually, it is achieved by a combination of home individual oral hygiene and regular professional care from a dentist or dental hygienist. Currently, in Slovakia, we are increasingly finding outpatient clinics where a dental hygienist works in symbiosis with a dentist. The study of the authors Woelber at al (2016) presents the results of the influence of a workshop in MI on non-surgical periodontal treatment performed by dental students. In the experimental group patients with periodontitis were treated by students trained in MI, while in the control group patients were treated by students who had not been trained in MI. There were 73 patients in the MI group and 99 patients in the control group. Regression analysis showed that there were no significant differences between groups with regard to plaque level, gingival bleeding, pocket depth reduction or bleeding upon probing. However, patients in the MIgroup showed significantly higher interdental cleaning self-efficacy than patients in the control group $(\mathrm{MI}=19.57 \pm 4.7$; control $=$ $17.38 \pm 6.01 ; p=0.016)$. Teaching MI to dental students resulted in a significant improvement in the self-efficacy of interdental cleaning in patients compared to a control group of non-trained students, but no improvement in other aspects of non-surgical periodontal therapy (20).

While oral hygiene remains the most important factor in obtaining and maintaining periodontal health, it should not remain the sole focus of attention. For adequate prevention and therapy, other factors such as ensuring treatment or maintaining clinically healthy periodontal tissues must be addressed. Rollnick et al (2007) presented a three-part (directing, guiding or following) model for healthcare clinicians to use when communicating with their patients in daily practice.

Current guidelines state that the clinician collaborates with the patient to help him/her identify his/her own goals and how he/she might best achieve them (15). 
Tab. 3. Algorithm of a patient to maintain periodontal health.

\begin{tabular}{l} 
1. Patient history \\
2. Examinations \\
3. Diagnosis \\
4. Preliminary plan of treatment \\
4.1 Preventive methods \\
4.2 Motivational interviewing \\
4.3 Health behavioural change tools \\
4.4 Communication style \\
4.5 Closing, summarizing, goal setting \\
5. Definitive plan of treatment \\
6. Conservative methods of treatment \\
7. Surgical methods of treatment \\
8. Supportive periodontal therapy \\
\hline
\end{tabular}

Motivational Interviewing (MI) is an evidence-based, patient-centered counseling approach for eliciting behavior change. The purpose of the study by Mills et al (2017). was to examine students' perceptions of the importance of MI and their confidence in applying it during patient care. Students' perceptions of importance increased with statistical significance in five out of eight MI strategies. Student perceptions of importance of using MI and their confidence in applying MI increased in a majority of the strategy categories. Successes with patient health behavior change and challenges with time to integrate this in practice were noted. Research on the longitudinal impact and faculty feedback calibration is recommended (22).

Applications of MI have spread far beyond clinical psychology into fields including health care, rehabilitation, public health, social work, dentistry, corrections, coaching, and education, directly impacting the lives of many people (23).

Gingival indices can reliably and objectively determine how the patient is involved in dental hygiene. We used a periodontal probe to measure characteristics that are central for future periodontal health. This critical issue is discussed in more details by Trombelli and Tatakis (24). Since we need to achieve results that are reproducible worldwide, the periodontal probe and its design are the focal point for measuring periodontal health. We use only plaque, gingival and periodontal indices that are known worldwide. This allows us to compare our own treatment results with those of dentists anywhere in the world and to compare treatment even in a patient for whom the individual investigators did not use the individual indices. We endeavour to proceed in terms of evidence-based dentistry procedures and procedures (Tab. 3).

To maintain periodontal health, a dentist must follow a preventive periodontal plan, which he realized gradually. Chronic infections such as periodontal disease may play a role in the development of cardiac disease and diabetes mellitus and are implicated in organ transplant rejection (25). The relevance of recognizing such important determinants of periodontal health and disease as controllable and uncontrollable predisposing and modifying factors cannot be underestimated, and their assessment for each patient is crucial to attaining and maintaining clinical periodontal health (2).

\section{Conclusion}

The definition of periodontal health is a crucial problem for dentists. Paradoxically, researchers and clinicians have been dealing with this definition in classification of periodontal disease and conditions only since 2018. Periodontal health must be controlled by every dentist in practice and in collaboration with the patients.

Periodontitis is a major public health problem due to its high prevalence, as well as because it may lead to tooth loss and disability, negatively affect chewing function and aesthetics, be a source of social inequality, and impair the quality of life (26). Consequences of periodontitis include substantial edentulism and masticatory dysfunction, resulting in significant dental care costs and a possible negative impact on general health.

Only a dentist who is able to diagnose periodontal health in a patient can be successful in managing more complex periodontological and implantological treatment.

However, before we begin diagnosing individual periodontal conditions and diseases, we must have absolutely perfect knowledge of what constitutes periodontal health. Only when we are in control of what the periodontal health of the patient entails we can continue to work on making a diagnosis and developing a treatment plan. Therefore, it appears to be reasonable in clinical concepts for periodontal care to (1) include assessments of patient behaviour and, if necessary, (2) apply effective behaviour change counselling methods (15).

Development of new generally accepted guidelines, such as periodontal health, motivational interviewing, adequate periodontal assessment and instructions in self-performed plaque control, and compliance with maintenance protocols appear to be the most important factors to limit or avoid potential negative effects on the periodontium.

There is still a proven rule that prevention is in this form better, simpler, more effective, and cheaper than the treatment of small or extensive destruction of the periodontal tissue of the tooth or the entire dentition of the patient.

\section{References}

1. World Health Organization. Constitution of WHO: Principles http:// www.who.int/about/mission/en. Accessed March 26, 2018.

2. Lang NP, Bartold PM. Periodontal health. J Clin Periodontol 2018; 45 (Suppl 20): S9-S16. https: //doi.org/10.1111/jcpe.12936

3. Eke PI, Dye BA, Wei L, Thornton-Evans GO, Genco RJ. Prevalence of periodontitis in adults in the United States. J Dent Res 2012; 91: 914-920

4. Eke PI, Dye BA., Wei L et al. Update on Prevalence of Periodontitis in Adults in the United States: NHANES 2009 to 2012. J Periodontol [online] 2015; 86 (5): 611-622. DOI: 10.1902/jop.2015.140520.

5. Murakami SB, Mealey E, Mariotti A et al. Dental plaque-induced gingival conditions. J Clin Periodontol [online] 2018; 45: S17-S27. DOI: 10.1111/jcpe.12937.

6. Zmora N, Bashiardes S, Levy M, Elinav E. The role of the immune system in metabolic health and disease. Cell Metab 2017; 25: 506-521.

7. Mariotti A, Hefti AF. Defining periodontal health. BMC Oral Health 2015; 15 (Suppl 1): S6. 
670-674

8. Caton J, Armitage G, Berglundh T et al. A new classification scheme for periodontal and periimplant diseases and conditions - Introduction and key changes from the 1999 classification. J Clin Periodontol 2018; 45 (Suppl 20): S1-S8. https: //doi.org/10.1111/jcpe.12935.

9. Tonetti MS, Eickholz P, Loos BG et al. Principles in prevention of periodontal diseases: consensus report of group 1 of the 11th European Workshop on Periodontology on Effective Prevention of Periodontal and Peri-Implant Diseases. J Clin Periodontol 2015; 42 (Suppl 16): S5-S11.

10. Chapple ILC, Mealey BL, Van Dyke T et al. Periodontal health and gingival diseases and conditions on an intact and a reduced periodontium: Consensus report of workgroup 1 of the 2017 World Workshop on the Classification of Periodontal and Peri-Implant Diseases and Conditions. J Clin Periodontol [online] 2018; 45: S68-S77. DOI: 10.1111/jcpe.12940.

11. Randall CL. On Motivational Interviewing for Oral Health Promotion: State of the Field and Future Directions. JDR Clin Translat Res [online] 2018; 3 (4): 376-377. DOI: 10.1177/2380084418796462.

12. Gumus F, Solak I, Eryilmaz MA. The effects of smoking on eutrophil/lymphocyte, platelet/ /lymphocyte ratios. Bratisl Med J 2018; 119 (2): 116-119.

13. Gao X, Lo ECM, Kot SCC et al. Motivational Interviewing in Improving Oral Health: A Systematic Review of Randomized Controlled Trials. J Periodontol [online] 2014; 85 (3): 426-437. DOI: 10.1902/ jop.2013.130205.

14. Curry-Chiu ME, Catley D, Voelker MA, Bray KK. Dental Hygienists' Experiences with Motivational Interviewing: A Qualitative Study. J Dent Educ 2015; 79 (8): 897-906.

15. Lindhe $\mathbf{J}$ et al. Clinical Periodontology and Implant Dentistry. Chichester: Wiley Blackwell, 2015.

16. Babjakova $\mathbf{J}$ et al. Risk behavioral survey in the sample of Slovak adolescents. Bratisl Med J 2019; 120 (12): 899 - 907.
17. Kann L, McManus T, Harris WA et al. Youth Risk Behavior Surveillance - United States, 2017. MMWR Surveill Summ 2018; 67 (8): 1-114.

18. Wilson AR, Fehringer KA, Henderson WG et al. Fidelity of motivational interviewing in an American Indian oral health intervention. Comm Dent Oral Epidemiol [online] 2018; 46 (3): 310-316. DOI: 10.1111/ cdoe. 12368.

19. Kay EJ, Vascott D Hocking A et al. Motivational interviewing in general dental practice: A review of the evidence. Brit Dent J [online] 2016; 221 (12): 785-791. DOI: 10.1038/sj.bdj.2016.952.

20. Woelber JP, Spann-Aloge N, Hanna G et al. Training of Dental Professionals in Motivational Interviewing can Heighten Interdental Cleaning Self-Efficacy in Periodontal Patients. Front Psychol [online] 2016; 7. DOI: 10.3389/fpsyg.2016.00254.

21. Rollnick S, Miller W, Butler C. Motivational Interviewing in Healthcare. New York: Guilford Press, 2007.

22. Mills A, Kerschbaum WE, Richards PS et al. Dental Hygiene Students ‘ Perceptions of Importance and Confidence in Applying Motivational Interviewing During Patient Care. Amer Dent Hygienists‘ Assoc 2017; 91 (1): 15-23.

23. Miller WR, Moyers TB. Motivational interviewing and the clinical science of Carl Rogers. J Consulting Clin Psychol [online] 2017; 85 (8): 757-766. DOI: 10.1037/ccp0000179.

24. Trombelli L, Farina R, Silva CO, Tatakis DN. Plaque-induced gingivitis: Case definition and diagnostic considerations. J Clin Periodontol 2018; 45 (Suppl 20): S44-S67.

25. Silvay G et al. Serious problem of oral health and dental evaluation before surgery. Bratislava Med J 2016; 117 (3): 185-187.

26. Papapanou PN, Sanz M et al. Periodontitis: Consensus report of Workgroup 2 of the 2017 World Workshop on the Classification of Periodontal and Peri-Implant Diseases and Conditions. J Clin Periodontol 2018; 45 (Suppl 20): S162-S170. https: //doi.org/10.1111/ jcpe.12946. 